

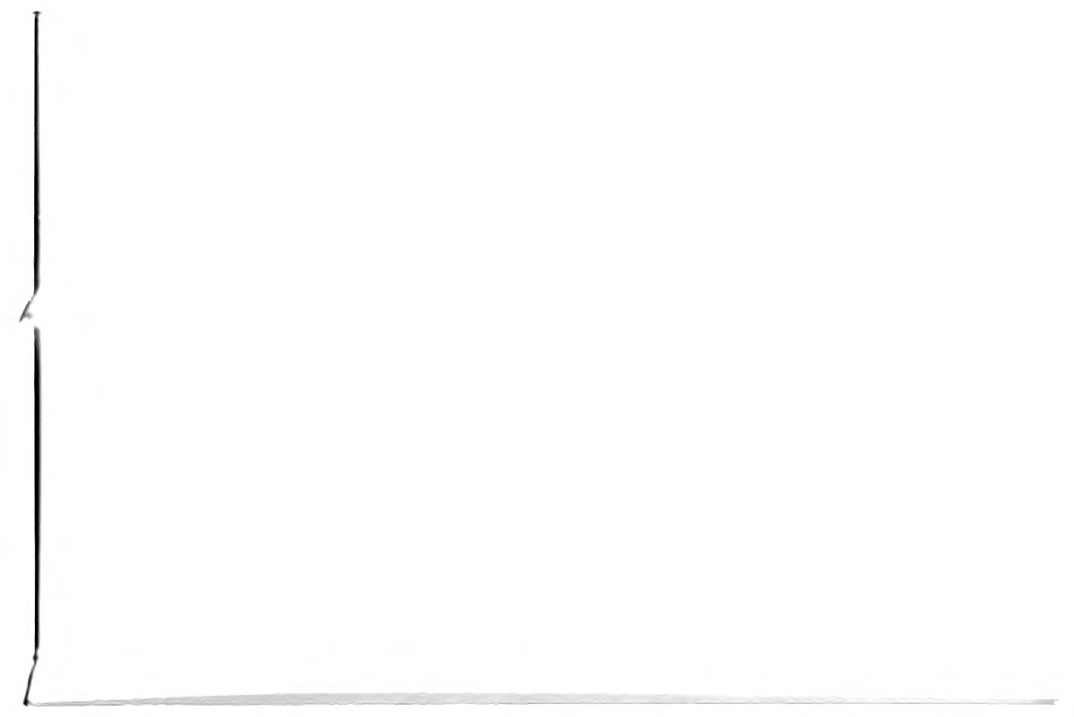


Constructive Hopf's theorem: or how to untangle closed planar curves

by

K. Melhorn, C. Yap

Technical Report No. 340

Robotics Report No. 134

January, 1988

\author{
New York University \\ Dept. of Computer Science
}

Courant Institute of Mathematical Scienccs

251 Mercer Street

New York, New York 10012

Work by the first author has been supported by DFG, Grant Me6-1. Work by the second author has been supported by NSF Grants \#DCR-84-01898 and \# DCR-84-01633. 


\title{
Constructive Hopf's theorem: or how to untangle closed planar curves
}

\author{
Kurt Meblhorn ${ }^{1}$ and Chee-Keng Yap ${ }^{2}$ \\ Angewandte Mathematik und Informatik \\ Courant Institute of Mathematical Sciences \\ Unjversitaet des Saarlandes \\ New York University \\ D.6600 Saarbruecken \\ 251, Mercer Street \\ Federal Republic of Germany \\ New York, NY 10012 \\ USA
}

\begin{abstract}
We consider the classification of polygons (i.e. closed polygonal paths) in which, essentially, two polygons are equivalent if one can be continously transformed into the otber without causing two adjacent edges to overlap at some moment. By a theorem of Hopf (for dimension 1, applied to polygons), this amounts to counting the winaing number of the polygons. We show that a quadratic number of elementary steps suffices to transform between any two equivalent polygons. Furthermore, this sequence of elementary steps, although quadratic in number, can be described and found in linear tinc. In order to get our constructions, we give a direct proof of Hopf's result.
\end{abstract}

November 13,1987

\footnotetext{
'Supported by DFG, Grant MeG-1

${ }^{2}$ Supported by NSF Grants \# DCR-84-01898 and \#DCR-84-01633
} 



\section{Why a circle differs from a figure-of-eight}

First consider closed planar curves that are smootb. It is intuitively clear that there are no smooth transformations from a figure-of-eight into a circle without introducing a 'kink' at some intermediate moment.
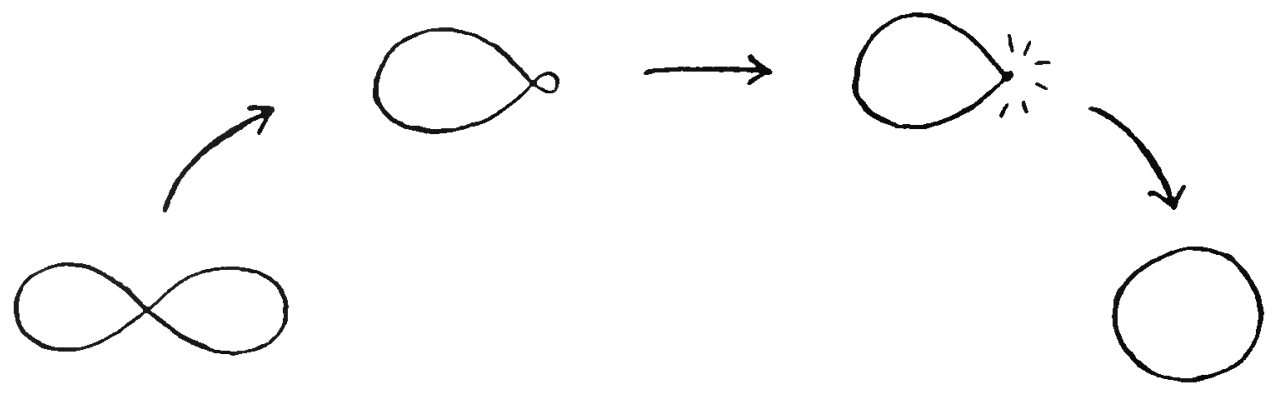

Figure 1. Appearance of a kink

We will say two closed planar curves are equivalent if one is transformable to the other in a kink-free manner and consider the problem of classifying the equivalence classes.

This problem has been completely solved, and we describe its mathematical formulation: let $C: S^{1} \rightarrow E^{2}$ be the natural parameterization (by arc-length) of a smooth closed curve, where $S^{1}$ is the unit circle. We then define $\omega_{C}: S^{1} \rightarrow S^{1}$ where $\omega_{C}(t)$ is defined to be the orientation of the dirccled tangent at the point $C(t)$. The notion of kink-free transformation is captured by saying that two curves $C$ and $D$ are equivalent if $\omega_{C}$ and $\omega_{D}$ are bonolopic. The winding number (or degree) of $C$ is defined to be

$$
\int_{S^{1}} d \omega_{C}
$$

For instance the winding number of the figure-of-eight and the circle are 0 and $2 \pi$, respectively. Then a famous theorem of Hopf (for dimension 1) says that $C$ and $D$ are equivalent if and only if they have the sanne winding number. See $[2,1]$.

The purpose of this paper is to give a constructive version of llop 's theorem for dimension one, in order to give quantitative and complexity information implicit in the result.

The rest of this paper is organized as follows. In section 2, we convert Hopf's theorem to the polygonal setting. In section 3 , we introduce a normal form for polygons. In section 4 , we give a constructive proof of Itopf's theorem for polygons. In scction 5 , we use the insights of the proof to develop a linear time algorithm. We conclude in section 6.

\section{Classification of Polygons}

In order to make our problem concrete (computational), we will turn this into a problem on closed polygonal patbs (which we will simply call a polygon). Since polygons bave kinks at their vertices, we reformulate kink-frce transformations as transformations of polygons that do not cause two adjacent edges to overlap at some point. This is made precise next. 
Deflnition: A path $\Pi$ is specified by a sequence

$$
\mathrm{II}=\left\langle v_{1}, v_{2}, \ldots, v_{n}>, n \geq 2\right.
$$

of points which we call vertices. The initial and final vertices are $v_{1}$ and $v_{n}$, respectively. We require $v_{i} \neq v_{i+1}$ for $i=1, \ldots, n-1$. The edges of the path are the line segments $\left[v_{i}, v_{i+1}\right]$ for $i=1, \ldots, n-1$. A closed path is one of the form

$$
\mathrm{II}=\left\langle v_{1}, \ldots, v_{n}, v_{n+1}>, n \geq 3\right.
$$

such that $v_{n+1}=v_{1}$. Two closed paths $\Pi, \Pi^{\prime}$ are said to combinatorially equivalent if one sequence can be obtained from the other by a cyclic shift, possibly followed by a reversal. More precisely, if $\Pi=\left\langle v_{1}, \ldots, v_{n}, v_{1}\right\rangle$, then $\Pi^{\prime}$ is either equal to

$$
\left\langle v_{i}, \ldots, v_{n}, v_{1}, \ldots, v_{i-1}, v_{i}\right\rangle
$$

for some $i=1, \ldots, n$, or the reverse of this sequence. A polygon $P$ on $n$ vertices is defined as the combinatorial equivalence class of some closed patb $\Pi=\left\langle v_{1}, \ldots, v_{n}, v_{1}\right\rangle$. We will express the polygon as

$$
P=\left(v_{1}, \ldots, v_{n}\right) \text {. }
$$

So $P$ can also be written as $\left(v_{2}, v_{3}, \ldots, v_{n}, v_{1}\right)$, say. For this paper, we further require that polygons satisfy the following local condition:

(C) For any consecutive triple $v_{i-1}, v_{i}, v_{i+1}$, if the three vertices are collincar, then $v$ lies strictly between the other two,

$$
v_{i}=\alpha v_{i-1}+(1-\alpha) v_{i+1}
$$

for some $0<\alpha<1$.

(Herc, as throughout the paper, arithmetic on indices of vertices of a polygon $P$ is modulo $n$, the length of the sequence $P$.) Note that (C) in particular prevents $v_{i}=v_{j}$ for $|i-j|=1$ or 2 . However, we allow $v_{i}=v$, for $|i-j|>2$ and in fact edges may even coincide. The reason for (C) is that the equivalence we seek allows local transformations that do not create kinks, and if (C) fails then it sometimes becomes ambiguous whether a kink is introduced.

The transformations we allow are of three types:

(T0) Inscrtion. We may transform $P=\left(v_{1}, \ldots, v_{n}\right)$ to

$$
Q=\left(v_{1}, \ldots, v_{i}, u, v_{i+1}, \ldots, v_{n}\right),(i=1, \ldots, n)
$$

where $u$ is a point lying strictly between $v_{i}$ and $v_{i+1}$.

(T1) Deletion. We may transform $P=\left(v_{1}, \ldots, v_{n}\right)$ to

$$
Q=\left(v_{1}, \ldots, v_{i-1}, v_{i+1}, \ldots, v_{n}\right),(i=1, \ldots, n)
$$


provided $v_{i-1}, v_{i}, v_{i+1}$ are collinear.

To introduce the last type of transformation, we need a definition. Relative to sny vertex $v_{i}$, we define two forbidden cones (at $v_{i-1}$ and $v_{i+1}$, respectively): the forbidden cone at $v_{i-1}$ is bounded by the two rays emanating from $v_{i-1}$, one ray directed towards $v_{i-2}$ and the otber directed away from $v_{i+1}$. Of the two choices of cones bounded by these rays, we choose the one that does not contain $v_{i}$. The forbidden cone at $v_{i+1}$ is similarly defined, being bounded by the two rays emanating from $v_{i+1}$ and directed towards $v_{i+2}$ and away from $v_{i-1}$, respectively. Each cone is a closed region so it includes the bounding rays. This definition applies for all $n \geq 3$. We are now ready for the third transformation type.
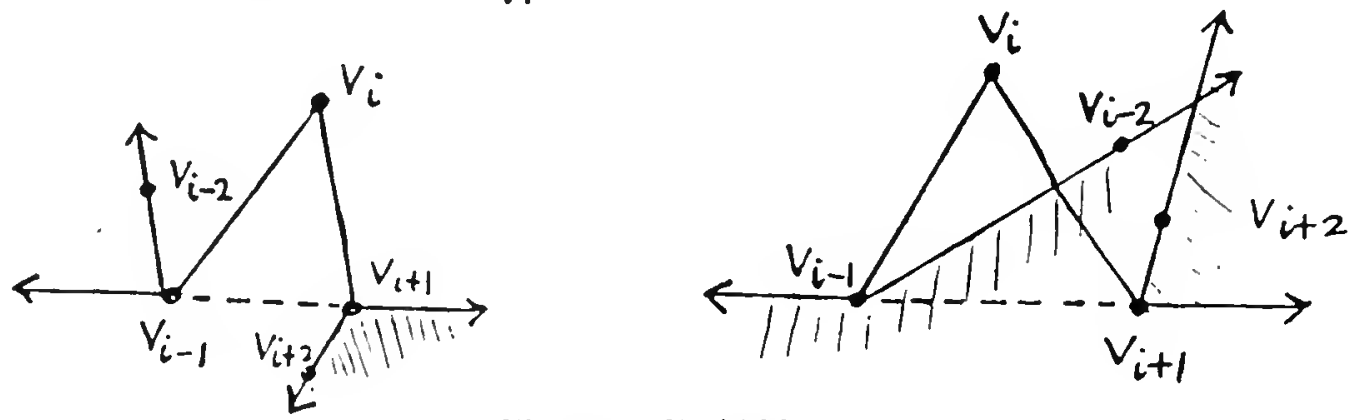

Figure 2. Forbidden cones

(T2) Translation. We may transform $P=\left(v_{1}, \ldots, v_{n}\right)$ to

$$
Q=\left(v_{1}, \ldots, v_{i-1}, u, v_{i+1}, \ldots, v_{n}\right),(i=1, \ldots, n)
$$

where $u$ is any noint not in the union of the two forbidden cones of $v_{i}$. In otherwords, we replace the point $v_{i}$ by $u$.

Definition: We say that two polygons $P, Q$ are equivalent if one can be transformed to the olber by a finite sequence of operations of types T0,T1,T2.

It is not bard to see that the relation is a true equivalence relation. Of course, we know from Hopf's theorem (applied to polygons) that it js sufficient to look at the winding number of a polygon. Ilowever, given two equivalent polygons $P$ and $Q$, it is unclear how one can

(A) transform $P$ into $Q$ by a sequence of (T0-T2) transformations,

(B) bound the number of transformation steps needed, and

(C) give an efficient algorithm to find a sequence of such transformation steps.

lndeed, standard proofs of Hopf's theorem yield no constructive information to answer these questions.

We answer these questions ( $A-C$ ) by defining a unique normal form (i.e. representative) of each equivalcnce class, and showing that a quadratic number of steps suffices to reduce a polygon into the normal form. The algorithm to find these steps runs in linear time. In particular, this solves the problem of transforming between two equivalent polygons $P, Q$ since the normal form is unique and the steps are reversible: first convert $P$ to the normal form and then reverse the steps from the normal form to $Q$. 
To think about what might be desirable, we nole (see Fig. 3) that the triangle and the bow-tie are obvious candidates for normal forms. Perhaps less convincingly, the 5-point star (5-8tar) also scems like a good candidate for a normal form.
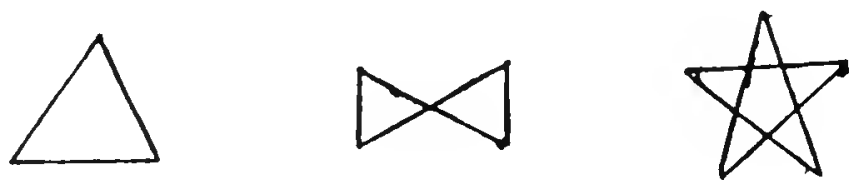

Figure 3. The triangle, bowtie and 5-star

The next Ggure illustrates a sequence of transformations to reduce a Victoria Cross polygon to one with fewer vertices.
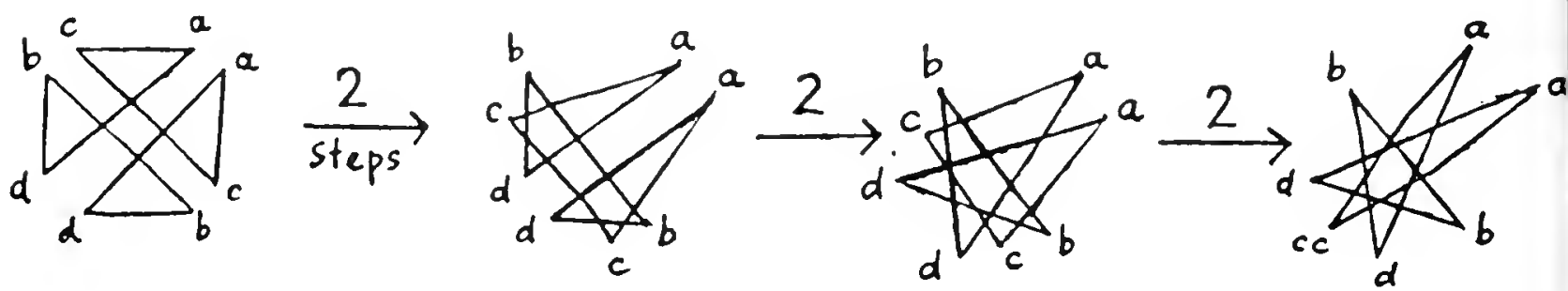

Figure 4. Reduction of the Victoria Cross

Remark. Any smooth closed curve can be approximated by a polygon and the transformations can discretized as a serics of our (T0-2) transformations. So in some sense we have also solved the corresponding question for smooth curves.

\section{Star Polygons}

Definition: $\Lambda$ polygon is reducible if it is equivalent to one with fewer vertices. It is irreducible otherwise.

One can check that the triangle, bow-tie and 5-star cannot be transformed by (T1) or (T2) transformations into any polygon with fewer vertices. But it turns out that even with (TO) transformations (which insert new vertices) the result is true: these are irreducible polygons. Since they have different number of vertices, it follows that they are inequivalent to each other. (Of course we can conclude this at once if we apply llopf's theorem.) In fact, all polygons on 3, 4 and 5 vertices are equivalent to these three candidates using only (T2) transformations. One may also check that there are no irreducible polygons on 6 vertices! 
The 5-star is also equivalent to the polygons in Fig. 5.
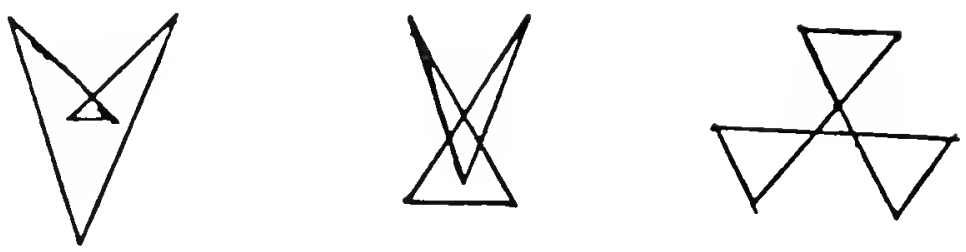

Figure 5. Polygons equivalent to the 5-star: the Fox, the Rabbit and Radioactive Sign

Note that the first polygon in Fig. 5 has the minimal number of self-intersections among its equivalence class, so perhaps it is a better choice of a normal form than the $\mathbf{5}$-star. This indicates that the choice of a good normal form is not obvious. Our first result, though simple, belps to narrow our choices considerably.

Theorem 1 Every polygon $P$ can be transformed by (T2) transformations into a polygon $Q$ all of whose vertices are distunct and lie on a circle.

Proof. Let $C$ be a circle that contains all the vertices of $F$ in its interior. For each vertex $v$ of $P$, we may move $v$ onto $C$ using a (T2) transformation: this follows from the observation that the non-forbidden region relative to $v$ always contain an infinite cone $K$ at $v$. We may move $v$ in any direction inside $K$ until we reach $C$. Furthermore, we can make sure that we avoid any vertex already on C. Q.E.D.

Henceforth, we assume that polygons have their vertices on some circle. Among the polygons on a circle, we define a particularly nice class.

Definition: $\Lambda$ patb II $=\left\langle v_{1}, \ldots, v_{n}\right\rangle$ is called a star path if the edges $e_{i}=\left[v_{i}, v_{i+1}\right]$ (for each $i=1, \ldots, n-1)$ intersect each of the edges

$$
e_{1}, e_{2}, \ldots, e_{i-1}
$$

Herc the edges are closed line segments and so $e_{i}(i \geq 2)$ always intersects $e_{i-1}$. A polygon $P=\left(v_{1}, \ldots, v_{n}\right)$ is called an $n$-star if for some choice of an initial vertex $v_{i}, i=1, \ldots, n$, the path

$$
\mathrm{II}_{i}=\left\langle v_{i}, \ldots, v_{n}, v_{1}, \ldots, v_{i-1}\right\rangle
$$

is a star path.

This terminology agrees with what we had called the 5-star before. The triangle is a 3-star and the bow-tie a 4-star. The following figure shows the next few n-stars. 

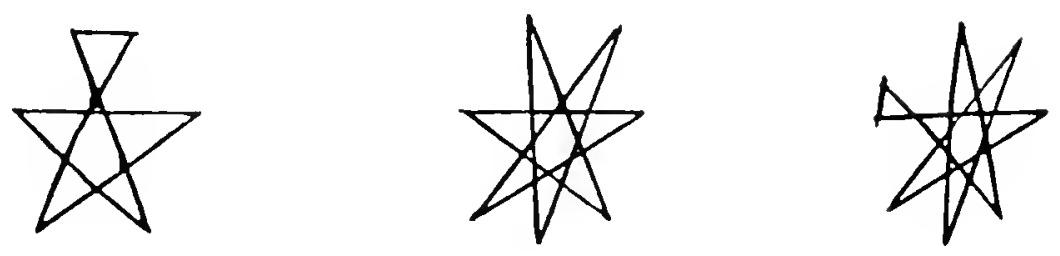

Figure 6. 6-, 7-, and 8-stars

The following lemma is easy:

\section{Lemma 2}

1. For $n=4$ or for odd values of $n$, if a polygon $P=\left(v_{1}, \ldots, v_{n}\right)$ is an $n$-star then for every choice of initial vertex $v_{i}$, the path $\Pi_{i}=\left\langle v_{i}, \ldots, v_{n}, v_{1}, \ldots, v_{i-1}\right\rangle$ is a star path. (In other words, the definition of a n-star does not depend on the choice of the initial vertex in these cases.)

2. For all other cases of $n$, there is a unique choice of an initial verlex $v_{i}$ which makes $\Pi_{1}$ a star path.

Lemma 3 Let $n, m$ be odd positive integers or equal to 1 . If $n \neq m$, then the $n$-star and the $m$-star are inequivalent.

Proof. One checks that the winding number of the 4-star is 0 and for each positive integer $k$, the $(2 k+1)$-star has winding number $\pm 2 k \pi$ (wbere the sign corresponds to a choice direction for the polygon). The result then follows from the fact that the winding number of a polygon is unchanged by any transformation of types (T0-2). Q.E.D.

This lemma supplies us with an infinite list of inequivalent polygons. We next prove that this list exhausts all the equivalence classes.

\section{A Canonical Form for Polygons}

We now set out to prove

Theorem 4 (Canonical Form) Every polygon can be transformed by a sequence of (T1) and (T2) transformations into an n-star, for some $n$ that is either odd or equal to 4 .

Corollary 5 An n-star is irreducible ij and only if $n=4$ or $n$ is odd.

Proof. Suppose that an $n$-star is irreducible. Then the theorem implies that $n$ must be 4 or odd. Conversely, let $n=4$ or odd. If an $n$-star is reducible, then the theorem shows that it is reducible to an $m$-star for some $m<n$ where $m=4$ or odd. This contradicts the previous lemma that the $n$ - and $m$-stars are inequivalent. Q.E.D. 
We prove the canonical form theorem by a sequence of lemmas.

A polygon that can (resp. cannot) be transformed to one with fewer vertices using just (T1) and (T2) transformations will be called semi-reducible (resp. semi-irreducible).

We need a useful notation. Henceforth, we write only the indices (i.e. subscripts) of vertices to denote the vertices. So we write $P=(1,2, \ldots, n)$ for $n \geq 3$. Next, if $u_{1}, u_{2}, \ldots, u_{k}(k \geq 3)$ are indices (not necessarily consecutive) we shall write

$$
u_{1}<u_{2}<\cdots<u_{k}
$$

to mean that as we traverse the circle of $P$ in a clockwise direction, starting from $u_{1}$, we will meet the indices $u_{1}, u_{2}, \ldots, u_{k}$ in this order.

The following simple fact is very useful:

Lemma 6 Suppose $P=(1, \ldots, n)(n \geq 4)$ is such that the pair of edges $[1,2]$ and $[3,4]$ do not intersect, and also the pair $[2,3]$ and $[4,5]$ do not intersect. Then $P$ is equivalent to $(1,2,4,5, \ldots, n)$ by a (T1) transformation. In other words, we may delete index $\$$.
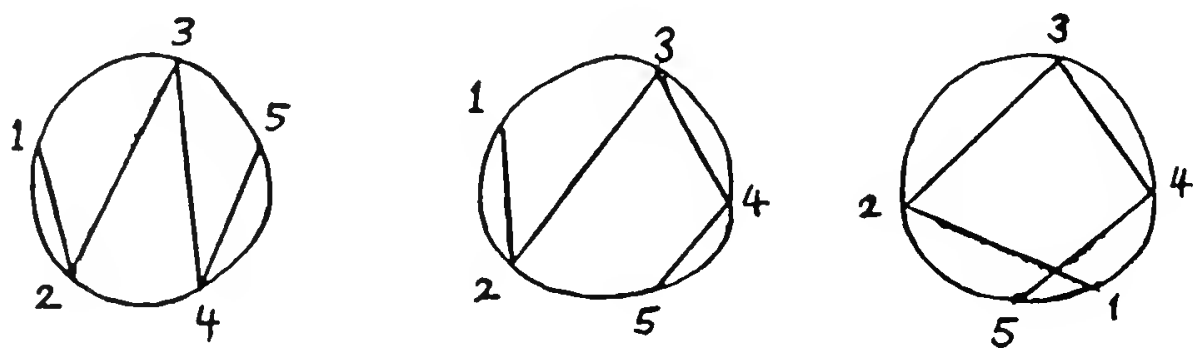

Figure 7. Can delete index 3

This is the only form of deletion of vertices used in our proof. Henceforth, whenever we delete vertices it is by appeal to this lemma.

We say that $P=(1,2, \ldots, n)$ contains an $N$-shape if $n \geq 4$ and for some choice of index $i$, we bave

$$
i<i+1<i+3<i+2
$$

or

$$
i<i+2<i+3<i+1
$$



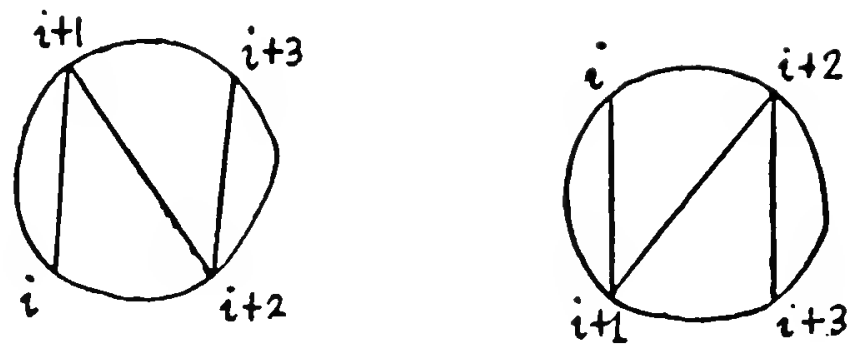

Figure 8. An N-shape

Lemma $T$ A semi-irreducible polygon $P=(1,2, \ldots, n)$ does not contain an $N$-shape unless $n=4$.

Proof. By way of contradiction, assume $P$ has an N-sbape. By symmetry, assume $1<2<4<3$.

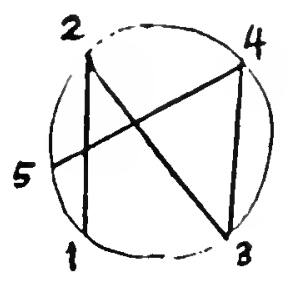

(a)

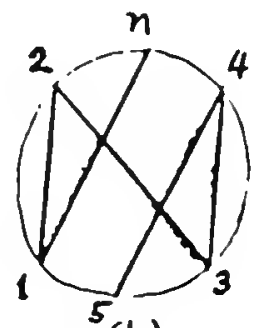

(b)

Figure 9. Reduction of an N-shape

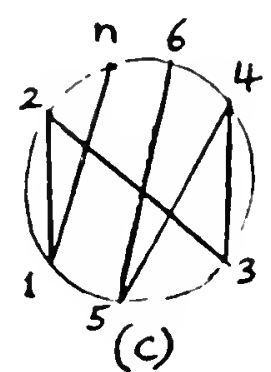

(c)

The result is true for $n=4$, so suppose $n \geq 5$. Since $P$ is semi-irreducible, by the previous lemma, the cdge $[4,5]$ must intersect $[2,3]$; hence $3<5<2$. Similarly, $2<n<3$. This shows that $n \neq 5$ so assume $n \geq 6$. If $1<5<2$ (Fig. 9a) then we can translate index 2 so that $1<2<5$ (this translation can occur because $2<n<3$ ). Then we can delete 3 . Therefore, we bave $3<5<1$. By symmetry, we bave $2<n<4$. The situation is shown in Fig. 9 b.

If $n=6$ then it is easy to see that $P$ is scmi-reducible. Otherwise, consider the location of index 6. There are two cases. First suppose $n<6<4$ (Fig. 9c). Then we may translate index 5 so that $2<5<6$. Then we can delcte index 3 . In the other case, $4<6<n$, we can translate index 4 so that $2<4<n$. Next translate index 3 so that $2<4<3<n$. Now we may delete index 2. Q.E.D.

Corollary 8 An n-star is semi-reducible if $n$ even and not equal to 1 .

Proof. Let $n$ be even, $n \neq 4$, and let $P=(1, \ldots, n)$ be an $n$-star. Then the vertices $n-2, n-1, n, 1$ forms an N-sbape. Q.E.D. 
We say that $P=(1,2, \ldots, n)$ conlains an $U$-shape if $n \geq 4$ and for some choice of index $i$, we have

$$
i<i+1<i+2<i+3
$$

or

$$
i<i+3<i+2<i+1 \text {. }
$$
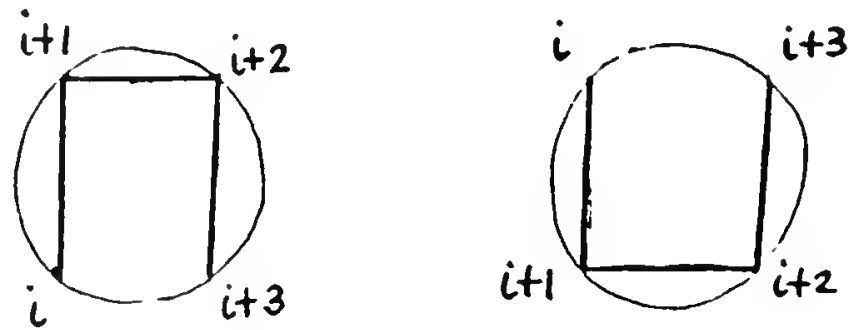

Figure 10. An U-sbape

Lemma 9 A semi-irreducible polygon $P=(1,2, \ldots, n)$ cannot contain a U.shape.

Proof. The result is true for $n=3,4$ and 5 , so assume $n \geq 6$. By the previous lemma, we know that $P$ does not contain an $\mathrm{N}$-shape. Suppose indices $(2,3,4,5)$ forms a $U$-shape as in the figure, $2<5<1<3$.

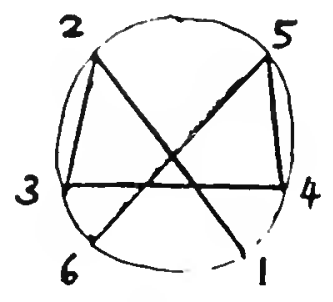

(a)

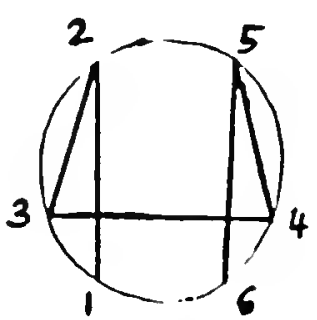

(b)

Figure 11. The elimination of U-shape

Since index 3 cannot be deleted, we have $4<1<3$, and similarly, since index 4 cannot be deleted, $1<6<3$. Suppose the relative position of indices 1 and 6 satisfies

$$
4<1<6<3
$$

as in Fig. 11a. Then we may translate index 3 so that $1<3<6$ and then translate index 4 so that $1<4<3<6$. Then we may delete index 3 (or 4 ), contradicting the semi-irreducibility of $P$. llence we may assume the situation of Fig. 1]b, with $4<6<1<3$.

Consider index $n$. If $2<n<1$ then $(n, 1,2,3)$ forms an $N$-shape which implies $P$ is semireducible. Hence we bave $1<n<2$. (In particular, this means $n \neq 6$, so $n \geq 7$.) So choose 
the smallest index $v$ from $7, \ldots, n$ such that $1<v<5$. Such a choice of $v$ exists since, a fortiori, $1<n<5$. We now use induction on $v$ to prove the following claim: we may transform $P$ such that indices $1, \ldots, 5$ remain fixed but index 6 satisfies

$$
4<1<6<3 .
$$

Clearly $v>6$. If $v=7$ then $(4,5,6,7)$ forms an $N$-shape which is a contradiction. If $v=8$ then note that $v-1<6<1<v$. We can translate 6 so that $1<6<v$; next translate 3 so that $1<6<3<v$. Now we may delete index 4 . Hence assume $v \geq 8$.

Consider indices $v-1, v-2$ and $v-3$. These vertices, by definition of $v$, all lie on the arc clockwise from index 5 to index 1 . Since there are no $N$-shapes in $P$, we have $v-1<v-2<1$. There are two cases for $v-3$. If $v-1<v-2<v-3$ then there are 2 possibilities for $v-4$ : either (a) $v-3<v-4<1$ or (b) $v-4<v-2<v-3$. If (a) holds then $v-2$ can be deleted. Hence (b) holds, in which case we may translate $v-3$ so that $1<v-3<v$. Now, for this transformed polygon, we can apply the induction hypothesis (replace $v$ by $v-3$ ). The other case for $v-3$ is $v-3<v-1<v-2$. Then we may translate $v-2$ so that $1<v-3<v$ and again we may apply the induction by pothesis (replace $v$ by $v-2$ ).

This completes the proof of the claim. But the claim transforms $P$ into the shape in Fig. 11a, which we already show is a contradiction. Q.E.D.

We now give the last lemma.

Lemma 10 Let $P=(1,2, \ldots, n)$ be any semi-jrreducible polygon. Then $I I=\langle 1,2, \ldots, n\rangle$ is a star path.

Proof. We now know that $P$ has no $N$ - and no U-shapes. We will show that $\Pi_{v}=\langle 1,2, \ldots, v\rangle$ $(v=3, \ldots, n-1)$ is a star path implies $\Pi_{v+1}$ is a star path. Consider the situation in Fig. 12 (so $1<3<2$ ).
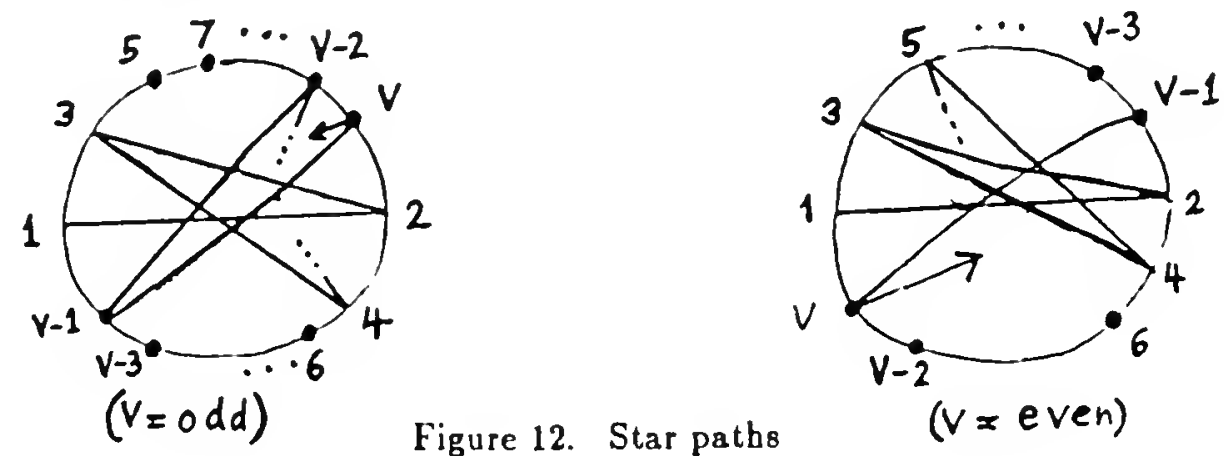

Figure 12. Star paths

The result $u=3$ follows from the previous two lemmas since if $\Pi_{4}$ is not a star path, then it forms a $\mathrm{U}$ - or an $\mathrm{N}$-shape. The same is true when $v=4$, so let $v \geq 5$.

First suppose $v$ is odd. If $I_{v+1}^{b}$ is not a star path then $1<v+1<v-2$. Then we can move index $v-1$ so that $1<v-1<3$. In fact, we can repeat this for $v-3, v-5, \ldots, 4$ (in this order) so that $1<v-1<v-3<\cdots<4<3$. Now $(1,2,3,4)$ is a U-shape, contradiction. 
Suppose $v$ is even. Then $2<v+1<v$ and we move indices $n-1, n-3, \ldots, 5$ (in this order) so that $1<5<7<\cdots<n-1<4$. Now $(2,3,4,5)$ is a U-shape. Q.E.D.

The main theorem now follows: given any polygon $P$, we can reduce it by (T1) and (T2) transformations until it is semi-irreducible. Then by the last lemma, the result must be an $n$-star. By the corollary to lemma $7, n$ cannot be even and not equal to 4. This proves the theorem.

\section{Algorithm}

The proof of the canonical form theorem contains an implicit quadratic time algoritbm to transform a polygon to its normal form. We now give a linear time algoritbm to construct the sequence of these quadratic transformation steps - this apparent paradox will be clarifed below.

Since transforming a polygon so that its vertices all lie on a circle is a linear time process, we may assume that the input polygon $P=\left(v_{1}, \ldots, v_{n}\right)$ is already in this form. We further assume that the circle is the unit circle centered at the origin, with $v_{1}=(-1,0)$ and $v_{2}=(1,0)$.

The algoritbm processes the input vertices in order. In the generic situation, the vertices $v_{1}, \ldots, v_{j-1}$ have been processed and the polygon bas been transformed into an equivalent polygon

$$
P_{j}=\left(u_{1}, \ldots, u_{i-1}, v_{j}, \ldots, v_{n}\right) \text {. }
$$

Furtbcrmore, we assume that

$$
\left\langle u_{1}, \ldots, u_{i-1}\right\rangle
$$

forms a star path. The current vertex being processed is $v_{j}$ although our algorithm may look ahead slightly, up to $v_{j+3}$. It is a realtime algorithm in the sense that each vertex takes $O(1)$ time to process, and it outputs $O(j)$ transformation steps with the processing of each vertex $v_{j}$. This apparent paradox (tbat $O(j)$ steps can be described in $O(1)$ processing time) arises because the $O(j)$ steps involve translating blocks of $O(j)$ vertices of a star path. Such transformations can be described in $O(1)$ time. Thus $P_{j}$ can be obtained from $P$ by applying the entire sequence of $O\left(j^{2}\right)$ transformation steps output up to now.

An interesting feature of the algorithrn is that it uses only $O(1)$ runtime memory. More precisely, at the moment of processing $v_{j}$, we only need in the active memory the values of the indices $i, j$; the values of the vertices

$$
v_{j}, \ldots, v_{j+3}
$$

and the sign (i.e. left or right) of the turns

$$
\left(u_{i-2}, u_{i-1}, v_{j}\right) \text { and }\left(u_{i-1}, v_{j}, v_{j+1}\right) \text {. }
$$

We will call the latter the sign information. In our algorithm, we must be careful to show bow we can reconstruct this sign information despite the fact that we do not explicitly store the $u$ 's.

For simplicity, in our description we will not explicitly say what (T1,T2) transformation steps will be output. But each step of the algorithm will be given justification and the reader can easily deduce the transformation steps needed. 
The relation $i \leq j$ always hold (since we do not create new vertices). Therefore it is unambigous to refer to the vertices by their indices, as long as it is clear whether an index is less than $i$ or at least $j$. We may now begin and assume that $i-1 \geq 3$ and $j \geq 4$. To initialize, we may let $u_{i}=v_{i}$ for $i=1,2,3$. Without loss of generality, let $1<3<2$ (i.e. $(1,2,3)$ is a left turn).

There are 4 cases to describe in the processing of vertex $v_{j}:\left(u_{i-2}, u_{i-1}, v_{j}\right)$ is either a left turn or a right turn, and $i$ is either odd or even. First assume $i=\operatorname{odd}$ (see Fig. AA).

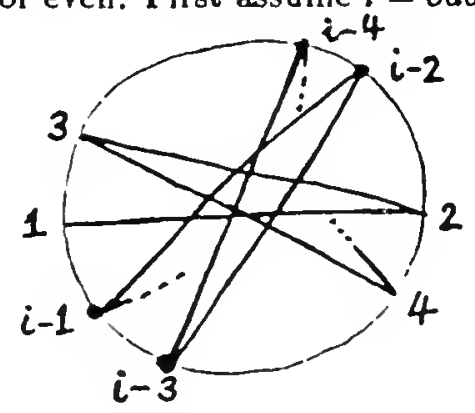

Figure AA. Case $i=$ odd

Case A. $(i-2, i-1, j)$ is a right turn.

Case A. $(i-1, j, j+1)$ is a left turn. Decrease $i$.

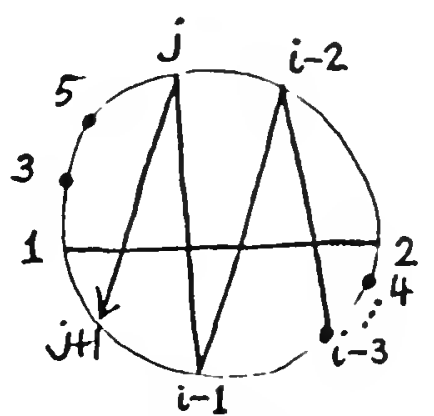

Figure BB. Case Al

Justification: See Fig. BB. We may delete $i-1$. To reconstruct the sign information, note that the lurns $(i-3, i-2, j)$ and $(i-2, j, j+1)$ are botb left turns.

Case A2. $(i-1, j, j+1)$ is a right turn. We bave some subcases.

Case A21. $j<j+1<2$. Decrement $i$ by 1 . 


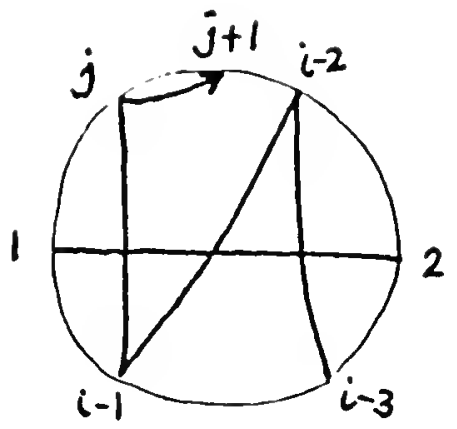

Figure CC. Case A21

Justification: We may assume that $j<j+1<i-2$ since we can move the odd $u$-vertices (i.e. $u_{3}, u_{5}, \ldots, u_{i-2}$ ) clockwise as close to index 2 as desired. ${ }^{3}$ This is shown in Fig. CC. We can then delete $i-1$ and note that $(i-3, i-2, j)$ and $(i-2, j, j+1)$ are left and right turns, respectively.

Case A22. $1<j<2<j+1$. Decrease $i$ by 1 .

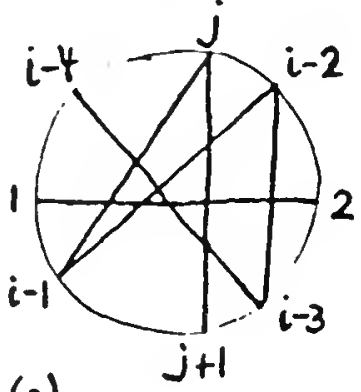

(a)

Figure DD. Case A22

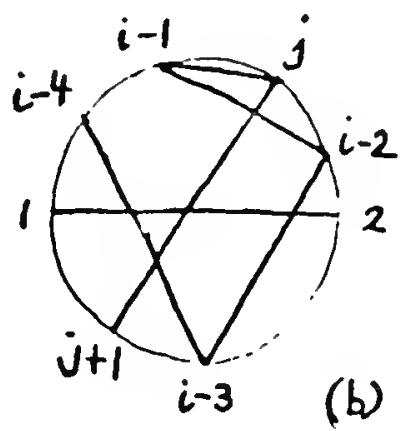

(b)

Justification: We may assume $i-4<j<i-2$ and $i-3<j+1<i-1$ since the u's can be moved appropriately. Then we are as in Fig. DD(a). We may now move $i-1$ such that $i-4<i-1<j$ (Fig. DD(b)), and then delete $i-2$. Now $(1, \ldots, i-3, i-1)$ is a star path and $(i-3, i-1, j)$ and $(i-1, j, j+1)$ are both right turns.

Case A23. $j<1<2<j+1$. So $(i-1, j, j+1)$ is a right turn (Fig. EE)

\footnotetext{
'Observe that this 'assuinption' actually require a sequence of $O(j)$ transformation steps to realize, but it is easily described in $O(1)$ time since we are transforming a consecutive sequence of vertices in atar path. This clarifies an easlier remark.
} 


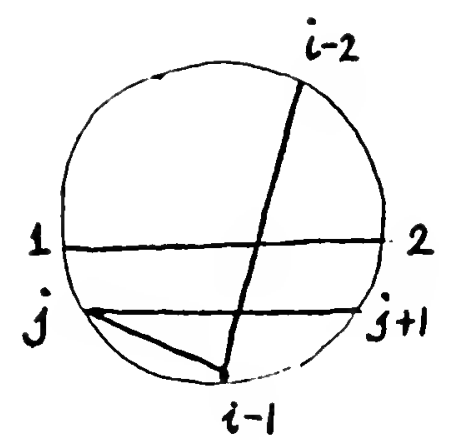

Figure EE. Case A23

Case A 231. $1 \leq j+2<j$. Decrease $i$ by 1 .

Justification: We can move $j$ such that $1<j<2$ which brings us into case A22.

Case A232. $j<j+2<1$. So $(j, j+1, j+2)$ is a left turn, see Fig. FF.

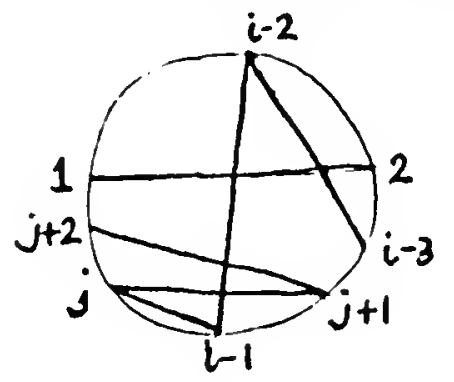

Figure FF. Case A232

Case A2321. $(j+1, j+2, j+3)$ is a right turn. Increment $j$ by 2 .

Justification: We may delete $j+1$ and $j$ (in that order). Also $(i-2, i-1, j+2)$ and $(i-1, j+$ $2, j+3)$ are both right turns.

Case A2322. $(j+1, j+2, j+3)$ is a left turn. Increment $j$ by 3 .

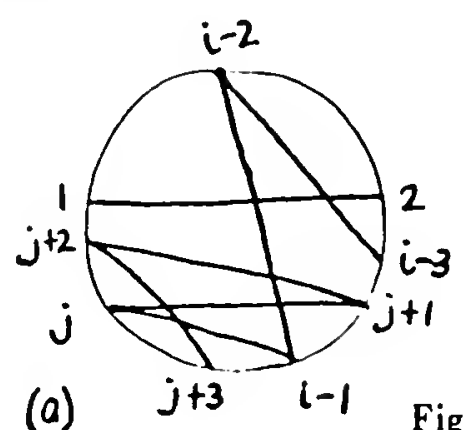

(a) $j+3 \quad i-1 \quad$ Figure GG. Case A2322

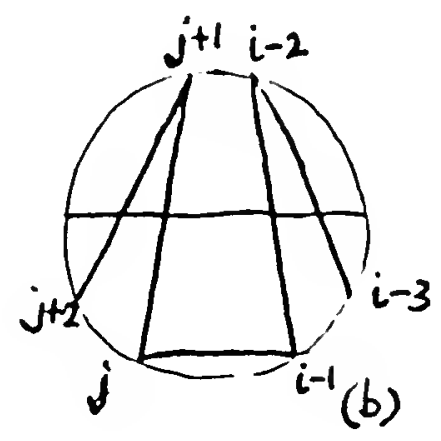

Juslificalion: See Fig. GG(a). We move $j+1$ counterclockwise so that $1<j+1<i-2$, as in Fig. $\mathrm{GG}(\mathrm{b})$. Now we may delete $i-1, j$ and $j+1$, in that order. Then $(1, \ldots, i-2, j+2)$ is a star 

path. Also $(i-2, j+2, j+3)$ is a left tura. We can determine the sign of the turn $(j+2, j+3, j+4)$
by looking at $j+4$.

Case B. $(i-2, i-1, j)$ is a left turn. Then $j \leq n$.

Cаве B1. $i-2<j<2$. Increment $i$ and $j$ by 1 each. See Fig. HH.

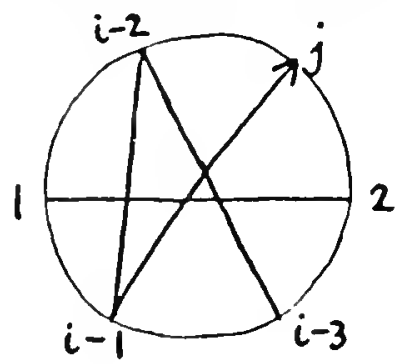

Figure HH. Case Bl

Justification: $(1, \ldots, i-1, j)$ is a star path.

Case B2. $2<j<i-1$.

Caвe B21. $(i-1, j, j+1)$ is a right turn. Increment $j$ by 1 .

Justification: By moving the even $u$-vertices (i.e. $u_{2}, \ldots, u_{i-3}$ ) if necessary, we may assume that $2<i-3<j$, as in Fig. JJ. Now delete $i-1$ and $(1, \ldots, i-2, j)$ is a star path. Also $(i-2, j, j+1)$ is a right turn.

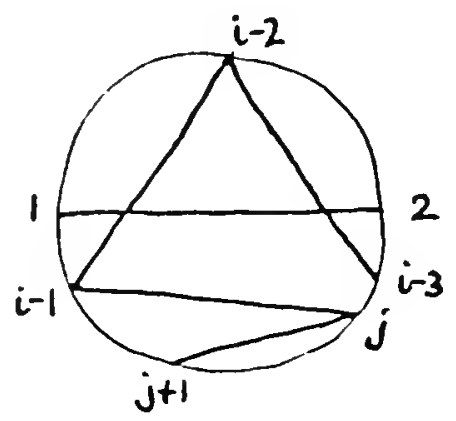

Figure JJ. Case B21

Case B22. $(i-1, j, j+1)$ is a left turn.

Cage B221. $j<j+1<1$. See Fig. KK 


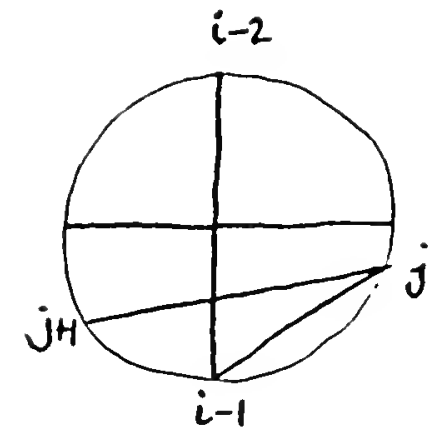

Figure KK. Case B221

Case B2211. $j<j+2<2$. Increment both $i$ and $j$ by 1 .

Justification: We can move $j$ counterclockwise so that $i-2<j<2$. This reduces to case Bl. Case B2212. $2<j+2<j$. See Fig. LL.

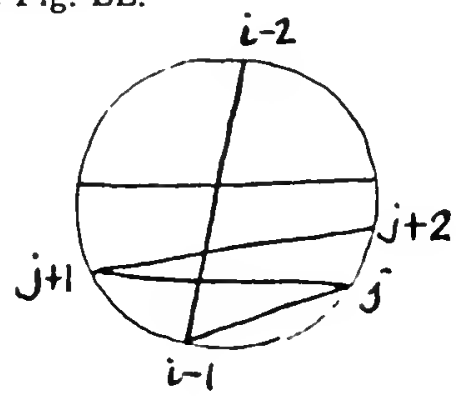

Figure LL. Case B2212

Cnse B22121. $(j+1, j+2, j+3)$ is a left turn. Increment $j$ by 2 .

Justification: We delete $j+1$ and then $j$. The turns $(i-2, i-1, j+2)$ and $(i-1, j+2, j+3)$ are botb left turns.

Case B22122. $(j+1, j+2, j+3)$ is a right turn. Increment $i$ by 1 and $j$ by 2 .

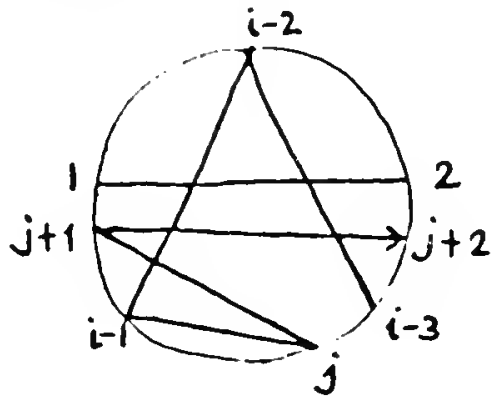

(a)
Figure MM. Case B22122

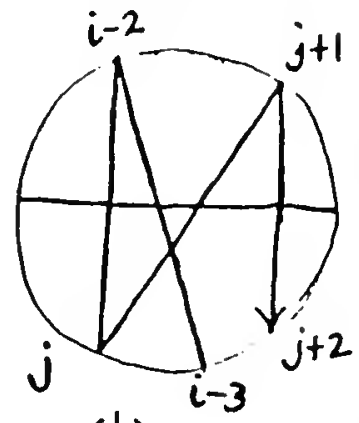

(b)

Justification: We may assume that $i-3<j<i-1$ (Fig. $\mathrm{MM}(\mathrm{a})$ ). Then we can move $j$ clockwise so that $i-2<j+1<2$ and delete $i-1$. The sequence $(1, \ldots, i-2, j, j+1)$ is a star 
path, Fig. MM(b).

Cage B222. $1<j+1<2$. Increment $i$ by 1 and $j$ by 2 .

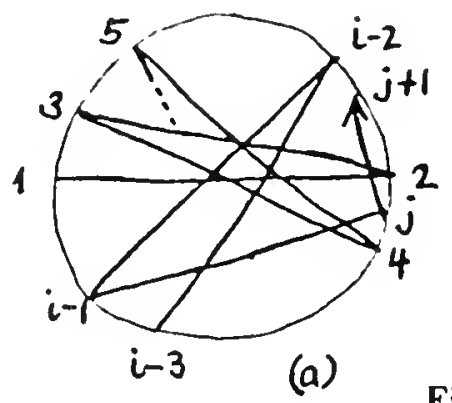

Figure NN. Case B222

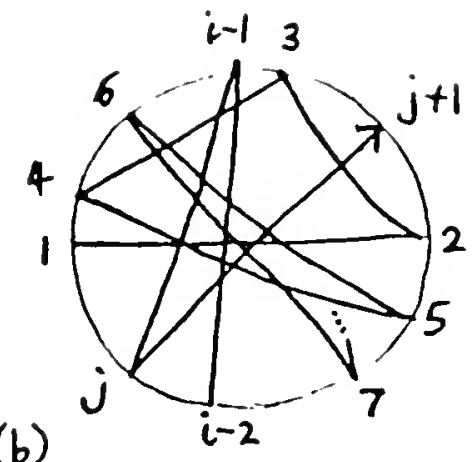

Justification: See Fig. NN(a). We may assume that $3<j+1<2$. Now move the vertices $i-2, i-4, \ldots, 7,5$ (in that order) clockwise between 2 and $j$, i.e.,

$$
2<5<7<\cdots<i-2<j
$$

Similarly move the vertices $i-1, i-3, \ldots, 6,4$ (in that order) clockwise between 1 and $j+1$, i.e.,

$$
1<4<6<\cdots<i-1<j+1 \text {. }
$$

See Fig. $N N(b)$. Now we delete index 3 and obverve that $(1,2,4,5, \ldots, i-1, j, j+1)$ is a star path. Obviously we can deduce the sign of the turns $(j, j+1, j+2)$ and $(j+1, j+2, j+3)$.

Case B223. $2<j+1<j$. Increase $j$ by 1 .

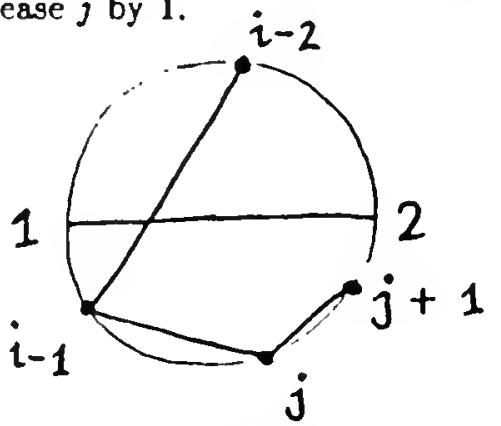

Figure OO. Case B223

Justification: We can delete $j$ in this situation. This completes subcase $\mathbf{B}$ and bence the case $i=o d d$. The case $i=$ even is similar and we leave it to the reader.

\section{Conclusion}

We have given an elementary proof of llopf's theorem, resulting in a linear algorithm to "untanglen any polygon. We empbasize that the true contribution of this work the construction of the 
transformation steps: checking if two polygons are equivalent is in itself a trivial process of keeping a cumulative sum of the angles turned. The key insight comes from putting the vertices on a circle, thercby reducing polygons to combinatorial objects (the cyclic permutation of their vertices around the circle).

Our proof shows incidentally: (1) It suffices to use (T1,T2) transformations to make a polygon irreducible and (2) any two equivalent irreducible polygons are inter-transformable using only (T2) transformations.

We can generalize the problem to the classification of polygonal paths. The transformations of vertices not near the endpoints are as before, with the following important modification: the transformation must not let an edge cross either endpoints of the path. Without this requirement, the classification becomes trivial. At the endpoints, we require that the end edges never overlap the imaginary edge connecting both endpoints. The methods of this paper ought to suffice for this classification.

We pose as a problem for further research to reduce the higher dimensional version of Hopf's theorem to a constructive, algorithmic form as we have done bere.

\section{References}

(1) H. Hopf. Abbildungaklassen n-dimensionaler mannigfaltigkeiten. Math. Annalen, 96:209-224, 1927.

[2] Solomon Lefscbetz. Introduction to Topology. Princelon University Press, 1949. 


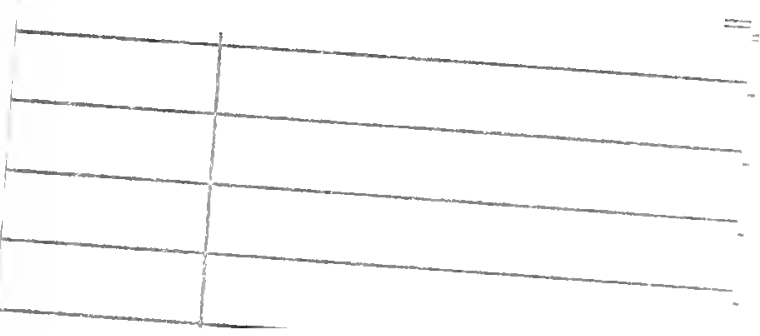

This hook may bo kept

\section{FOURTEEN DAYS}

A finc will be charged for each day the brok is kept overtire.

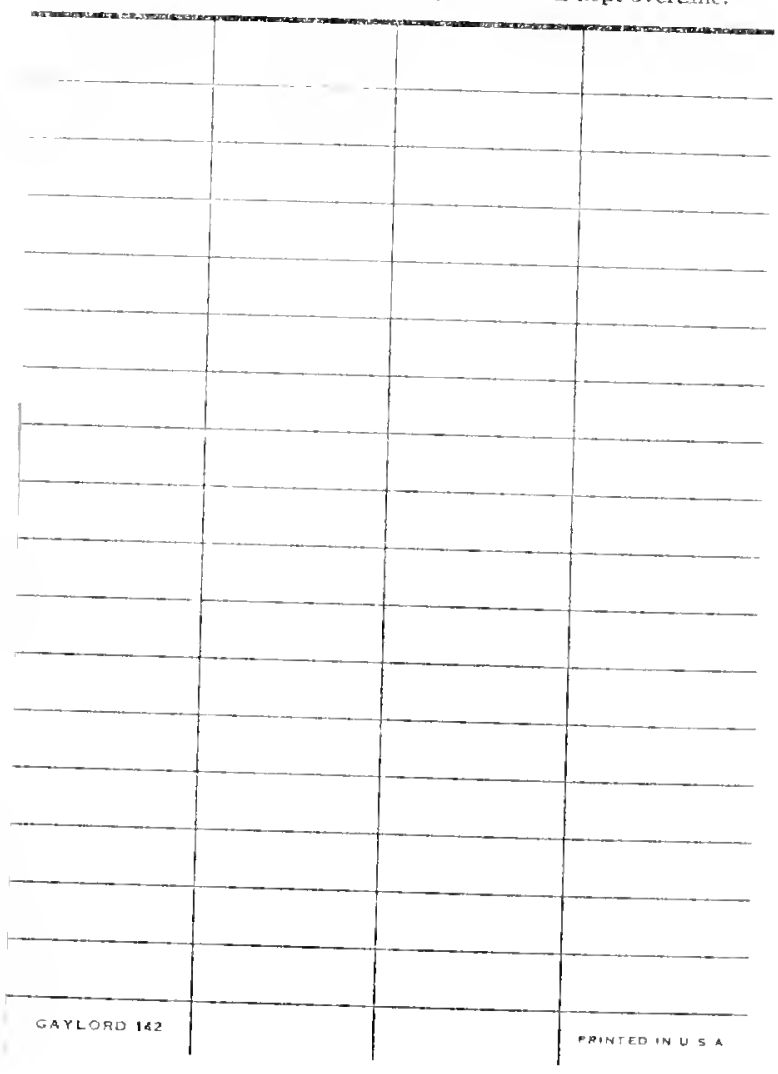


sw

nater

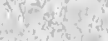

is

and

a

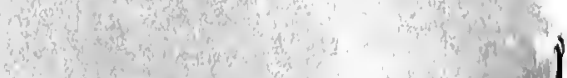

